\title{
Fundamental principles of an anti-VEGF treatment regimen: optimal application of intravitreal anti-vascular endothelial growth factor therapy of macular diseases
}

\author{
Paolo Lanzetta ${ }^{1}$ - Anat Loewenstein ${ }^{2}$ • The Vision Academy Steering Committee
}

Received: 5 October 2016 / Revised: 22 January 2017 / Accepted: 20 March 2017 / Published online: 19 May 2017

(C) The Author(s) 2017. This article is an open access publication

\begin{abstract}
Background Intravitreal anti-vascular endothelial growth factor (VEGF) therapy is now considered the gold standard for the treatment of various retinal disorders. As therapy has evolved, so too have the treatment regimens employed by physicians in clinical practice; however, visual outcomes observed in the real world have typically not reflected those reported in clinical trials. Possible reasons for this include a lack of consensus on treatment regimens and a lack of clarity about what the aims of treatment should be.

Methods The Vision Academy Steering Committee met to discuss the principles of an ideal treatment regimen, using evidence from the literature to substantiate each point. Literature searches were performed using the MEDLINE/PubMed database (cut-off date: March 2016) and restricted to English-language publications. Studies with fewer than ten patients were excluded from this review.

Results The Steering Committee identified the following four key principles for the ideal treatment regimen for anti-VEGF management of retinal diseases:
\end{abstract}

1. Maximize and maintain visual acuity (VA) benefits for all patients

Paolo Lanzetta and Anat Loewenstein are also members of the Vision Academy Steering Committee (all members are listed in the Appendix)

Paolo Lanzetta

paolo.lanzetta@uniud.it

1 Department of Medicine - Ophthalmology, University of Udine, Piazzale S. Maria della Misericordia, 33100 Udine, Italy

2 Tel Aviv Sourasky Medical Center, Tel Aviv, Israel
2. Decide when to treat next, rather than whether to treat now

3. Titrate the treatment intervals to match patients' needs

4. Treat at each monitoring visit.

Conclusions It is proposed that the adoption of a proactive and more personalized approach in the clinic such as a treatand-extend regimen will lead to benefits for both the patient and the physician, through a reduction in the associated treatment burden and better utilization of clinic resources. Implementation of the four principles should also lead to better VA outcomes for each patient, with a minimized risk of vision loss.

Keywords Anti-vascular endothelial growth factor · Retinal disease $\cdot$ Treatment regimens - Visual acuity · Aflibercept . Treat-and-extend

\section{Introduction}

The Vision Academy is a global group of ophthalmic experts whose goal is to identify and address unmet needs in ophthalmology. The Vision Academy Steering Committee (see Appendix) met in 2014 to discuss the principles of an ideal treatment regimen. This review documents the key points from that discussion, and provides evidence from the literature to support these recommendations. These principles were not conceived as a specific set of guidelines or an in-depth review of treatment regimens (which have been covered elsewhere [1]), but rather as a framework to encourage discussions between retinal specialists on how to achieve optimal outcomes for each patient while optimizing treatment and clinical practices. 


\section{Anti-VEGF treatment of retinal diseases}

Retinal disorders, including neovascular age-related macular degeneration (nAMD), macular edema following branch and central retinal vein occlusion (BRVO and CRVO), myopic choroidal neovascularization $(\mathrm{mCNV})$ and diabetic macular edema (DME), are major causes of preventable vision loss and impairment worldwide [2-4].

AMD is the leading cause of blindness in the elderly [2] and affects $10-13 \%$ of adults aged 65 years or over in North America [5]. It is predicted that by 2020, approximately 196 million people will be living with this disease, as a consequence of an aging population [6]. In 2014, the worldwide prevalence of DME was estimated to be around 21 million [4], with this figure expected to rise to 100 million by 2030 [7], due to an increase in the incidence of diabetes. This in turn is due to a number of factors, particularly an aging and increasingly obese population. As the onset of diabetes and subsequent complications is most common in young or middle-aged groups, the disease affects patients during their productive working lives [4]. Visual impairment on a global scale thus translates into a significant economic burden, including direct costs of treatment, other direct costs associated with vision impairment (e.g., nursing home costs and guide dogs), and billions of dollars in lost productivity each year due to a reduced labor force [8].

Some of the early treatment options for these conditions, such as laser photocoagulation and photodynamic therapy (PDT), have since been associated with poor visual outcomes and risk of adverse effects [9-17]. While these may still have a limited role in the management of retinal disorders, the advent of intravitreal therapy specifically targeting VEGF-A has revolutionized the treatment of such disorders, providing rapid and sustained improvements in VA alongside favorable sideeffect profiles.

Pegaptanib sodium $\left(\right.$ Macugen $^{\circledR}$ ), the first anti-VEGF agent to be approved for the treatment of nAMD [13], was designed to specifically target the VEGF-A 165 isoform [18-20]. However, experience has shown pegaptanib sodium to be less effective at improving visual outcomes than newer anti-VEGF agents that target all VEGF-A isoforms [21].

Bevacizumab (Avastin ${ }^{\circledR}$ ) and ranibizumab $\left(\right.$ Lucentis $\left.^{\circledR}\right)$ are anti-VEGF agents that are used more frequently than pegaptanib. Bevacizumab is licensed for use in several adult cancers [13], but has been used off-label for nAMD treatment since 2005 [22]. The absence of routine pharmacovigilance and the general risks of compounding and off-label use of medications not intended for ocular use all add to the still partially unknown safety profile of bevacizumab for the treatment of retinal disorders, when compared with licensed antiVEGF agents [23].

Ranibizumab has been licensed since 2006 for the treatment of nAMD [13], based on the efficacy demonstrated in the key trials, ANCHOR and MARINA, which were the first Phase III trials to show improvements in VA outcomes for all subtypes of CNV secondary to AMD [10, 17, 24]. Subsequent clinical trials, including BRAVO, CRUISE, RISE, and RIDE, demonstrated the efficacy of ranibizumab on a fixed, monthly dosing regimen across multiple indications $[10,11,17$, 24-30].

In some instances, the efficacy of both the $0.3 \mathrm{mg}$ and $0.5 \mathrm{mg}$ doses were compared when administered on a monthly regimen. In ANCHOR, VA outcomes at 24 months with monthly $0.3 \mathrm{mg}$ ranibizumab versus monthly $0.5 \mathrm{mg}$ ranibizumab were +8.1 and +10.7 Early Treatment Diabetic Retinopathy Study (ETDRS) letters respectively [11]. In MARINA, VA outcomes at 24 months with monthly $0.3 \mathrm{mg}$ ranibizumab versus monthly $0.5 \mathrm{mg}$ ranibizumab were +5.4 and +6.6 ETDRS letters respectively [17]. In BRAVO, which investigated the efficacy of ranibizumab in patients with macular edema following BRVO, the mean changes in bestcorrected visual acuity (BCVA) from baseline to month 6 were 16.6 and 18.3 letters in the $0.3 \mathrm{mg}$ and $0.5 \mathrm{mg}$ groups respectively [31]. Similarly, in CRUISE, which investigated the efficacy of ranibizumab in patients with macular edema following CRVO, the mean changes in BCVA from baseline to month 6 were 12.7 and 14.9 ETDRS letters in the groups treated with $0.3 \mathrm{mg}$ or $0.5 \mathrm{mg}$ ranibizumab respectively [32]. In a pooled analysis of RISE and RIDE, 36-month VA outcomes in patients with visual impairment due to DME were +12.4 and +11.2 ETDRS letters with the $0.3 \mathrm{mg}$ monthly dose and the $0.5 \mathrm{mg}$ monthly dose respectively [29]. These studies highlight the comparable efficacy of the two doses when administered on a monthly regimen.

Following on from this, the European registration trial for ranibizumab in DME, RESTORE, focused on the $0.5 \mathrm{mg}$ dose, but administered via a pro re nata (PRN) regimen, following three monthly loading doses [33]. The BCVA at the primary endpoint at 12 months was 6.1 ETDRS letters in the group randomized to ranibizumab from the beginning, and was 8.0 letters at 36 months (following the 2-year extension study) [34].

Intravitreal aflibercept $\left(\right.$ EYLEA $\left.^{\circledR}\right)$ was licensed for the treatment of nAMD in 2011 [13]. As well as inhibiting all isoforms of VEGF-A, aflibercept binds and inhibits VEGF$\mathrm{B}$ and placental growth factor [35-37], and may also have other potential targets [38]. The VIEW trials demonstrated that in patients with nAMD, three initial monthly doses of intravitreal aflibercept $2.0 \mathrm{mg}$ followed by dosing every 2 months delivered clinically equivalent outcomes to monthly ranibizumab $(0.5 \mathrm{mg})$, but with five fewer injections in year 1 [39]. From weeks 52 through to 96 , patients received their original drug assignment via a PRN-type regimen, in which therapy was administered as needed, with defined retreatment criteria and mandatory dosing at least every 12 weeks [40]. By week 96, the mean change in BCVA from baseline was a gain 
of 7.6 ETDRS letters in the groups previously assigned to intravitreal aflibercept dosed either monthly or every 2 months (bimonthly).

Intravitreal aflibercept is also licensed for the treatment of visual impairment due to $\mathrm{mCNV}$, and due to macular edema secondary to DME or RVO. The VIBRANT, COPERNICUS, and GALILEO clinical trials all demonstrated the efficacy of intravitreal aflibercept in patients with macular edema following BRVO and CRVO, and showed that treatment with intravitreal aflibercept resulted in rapid vision gains of 12-13 letters after the first injection alone, with these gains maintained for at least 1 year [41-44]. Similar gains were also demonstrated in the MYRROR study in mCNV, where patients treated with intravitreal aflibercept were gaining an average of 13.5 letters by week 48 [45].

The VIVID and VISTA studies investigated the efficacy of intravitreal aflibercept in patients with DME. Not only were gains in vision maintained to week 100 (e.g., in VISTA, mean gains in BCVA from baseline to week 100 were 11.5 and 11.1 letters, with intravitreal aflibercept dosed monthly or bimonthly respectively [46]), but signs of diabetic retinopathy also regressed in patients treated with intravitreal aflibercept [46, 47].

A head-to-head comparison of anti-VEGF agents in the treatment of DME was conducted by the Diabetic Retinopathy Clinical Research Network [48, 49]. Using strict optical coherence tomography (OCT)- and visiondriven monthly retreatment criteria, the mean gains in VA from baseline to the primary endpoint at 12 months were $13.3,11.2$, and 9.7 with intravitreal aflibercept, ranibizumab, and bevacizumab respectively $(p<0.001$ for intravitreal aflibercept versus bevacizumab, and $p=$ 0.031 for intravitreal aflibercept versus ranibizumab) [49]. In particular, intravitreal aflibercept treatment was associated with numerically better outcomes in patients with a baseline VA of less than 69 ETDRS letters, with statistically significant results for intravitreal aflibercept ( $p<0.001$ for intravitreal aflibercept versus bevacizumab, and $p=0.003$ for intravitreal aflibercept versus ranibizumab). This positive trend continued to 24 months, where VA outcomes in this subgroup of patients were 18.1, 16.1, and 13.3 letters, with intravitreal aflibercept, ranibizumab, and bevacizumab respectively ( $p=0.02$ for intravitreal aflibercept versus bevacizumab, and $p=0.18$ for intravitreal aflibercept versus ranibizumab) [48].

Each anti-VEGF agent is likely to require a specifically optimized treatment regimen, but this must be balanced against the practicalities and costs of implementing each regimen in the clinic. Anti-VEGF agents have now been in ophthalmic use for the past 10 years [17], and the wealth of data from clinical and real-world studies support the clear, positive benefit-risk balance associated with their use for treatment of retinal disorders $[50,51]$.

\section{Anti-VEGF therapy in clinical practice}

In each indication (excluding $\mathrm{mCNV}$ ), the best outcomes have been shown in the clinical trials that employ fixed dosing [39]. Such a regimen is predictable and therefore straightforward for both the clinic and the patient. However, these regimens are usually associated with high clinic and patient burden (especially with monthly visits), and with risks of either over- or under-treatment if the fixed intervals between treatments are too short or too long [52]. For instance, the EXCITE study investigated the efficacy of ranibizumab $0.3 \mathrm{mg}$ dosed on a monthly or quarterly regimen, following a loading dose of three consecutive monthly injections [52]. At month 12, the mean changes in BCVA were 8.3 and 4.9 ETDRS letters with the monthly and the quarterly doses respectively.

Numerous studies (especially with ranibizumab) have shown the challenges of bringing the efficacy demonstrated in clinical trials into the real world [53-57]. Given that antiVEGF therapies for the treatment of DME were only approved in 2012, robust studies on the real-world effectiveness of anti-VEGF therapies for this indication have not yet been published. Nevertheless, translating the outcome achieved with fixed dosing in clinical trials will be difficult in clinical practice. Clinical practice has often been a compromise; fixed, monthly dosing is associated with huge burdens for both the patient and the clinic. Therefore, in real-world practice, PRN, treat-and-extend, and other regimens are adopted.

In PRN-type regimens, patients are only treated on disease reactivation, in an effort to reduce injection frequency and costs. Patients typically follow a monthly schedule of clinic visits, where the decision to re-treat at each visit is based on a set of prespecified criteria, as determined by the physician (e.g., VA and OCT/fluorescein angiography assessments) $[58,59]$. Outcomes obtained with this regimen depend largely on the retreatment criteria employed by the treating physician and how closely these are adhered to. For example, Protocol T, which employed the use of strict retreatment criteria, saw favorable outcomes for ranibizumab and intravitreal aflibercept at the end of year 1 in patients with DME [49], and highlights the need to define, interpret, and adhere to retreatment criteria. RESTORE, however, which used predominantly VA-based retreatment criteria, resulted in substantially less VA gain at the end of year 1 [33]. Although cross-trial comparison is difficult due to differences in patient characteristics, it should be noted that even in a clinical trial setting some physicians will not adhere to the retreatment criteria, resulting in undertreatment of patients.

A treat-and-extend regimen is a more proactive and customized approach to treatment, with the goal of preventing disease recurrence. The drug is administered at every scheduled visit, regardless of visual or anatomic status on the day. However, the interval between each visit is either increased or decreased according to the anatomic and VA status, to 
determine the maximum time between injections without disease recurrence, i.e., the maximum recurrence-free interval (Fig. 1 gives an illustrative example of how a treat-andextend regimen might be implemented in the clinic). The interval between treatments is then kept at a slightly shorter duration than that of the maximum recurrence-free interval to minimize the possibility of disease recurrence $[13,60]$ and maximize the length of time the patient receives protection from the drug. This approach reduces the frequency of clinic visits and removes the requirement for interim monitoring between injections, which helps to make the disease more manageable for the patient and physician while still ensuring that an appropriate number of injections are administered. This may also reduce the anxiety experienced by patients who fear disease recurrence, as well as any uncertainty regarding whether they will receive an injection at their forthcoming appointment [61]. The psychological and physical impact of any treatment regimen on patients is a factor that should always be considered.

The discrepancy in outcomes between clinical trials and the available real-world data is probably due to a number of factors.

Most physicians face organizational challenges related to clinic flow and follow-up frequency, usually due to resource limitations (including staff, VA assessors, and OCT machines). Reimbursement is also an issue, with some authorities imposing complicated approval procedures or limitations on patient characteristics.

Patient adherence to treatment regimens is another critical element. Although PRN regimens may help to reduce the number of treatments, many patients struggle to adhere to the necessary monthly monitoring schedule, which can result in wasted clinic resources. Patient adherence is itself affected by several factors, including age, psychological status, expectations of treatment, ease of travel, and cost (particularly where patients bear a large portion of the cost of treatment themselves).

\section{Methods}

The Vision Academy Steering Committee met to discuss and define the focus of an ideal treatment regimen, and consider what would need to be done to help achieve this, if freed from the constraints of resource limitations or practical barriers. The practiced regimens and the associated advantages and disadvantages of each were initially reviewed. Data were examined from clinical trials and real-world studies, focusing on the licensed anti-VEGF agents used for the treatment of visual impairment secondary to nAMD, DME, and RVO. Literature searches were performed using the MEDLINE/ PubMed database (cut-off date: March 2016) and restricted to English-language publications. To ensure a high level of evidence from the included data, studies with fewer than ten patients were excluded.

\section{Results: the principles of an ideal treatment regimen}

Following a round-table discussion where the merits of each principle were considered, the Steering Committee consensus was that an ideal treatment regimen should encompass the following four principles:

1. Maximize and maintain VA benefits for all patients

2. Decide when to treat next, rather than whether to treat now

3. Titrate the treatment intervals to match patients' needs

4. Treat at each monitoring visit.

These principles were developed with consideration of chronic retinal diseases that require regular therapy, as opposed to those diseases requiring less frequent treatment and less rigorous management (e.g., treatment of $\mathrm{mCNV}[27,45]$ ).

\section{Maximize and maintain VA benefits for all patients}

Maximizing and maintaining VA for all patients should be a fundamental principle of any treatment regimen. The impact of improved and maximized VA on quality of life for patients is an important consideration, with improvements in BCVA shown to have a significant effect on a patient's functional abilities [62]. A 5-letter increase in VA in the best-seeing eye has been shown to nearly double the likelihood of a patient being able to read a newspaper, and drive at night or under difficult conditions [62]. The significance of achieving driving vision for patients should not be underestimated; driving ability is often seen as an important measure of independence [63-65].

Clinical trials demonstrate that significant gains in VA are possible, and that these can be maintained in the long term. This is true across all key intravitreal aflibercept trials, including VIEW 1 and 2 [40, 66], COPERNICUS and GALILEO [42, 43], VIBRANT [67], and VIVID and VISTA [46]. In these studies, VA gains with intravitreal aflibercept were maintained over at least 1 year, and in some instances, for 4 years [66] (manuscript in development). Similar outcomes were also seen across the key ranibizumab trials, including ANCHOR and MARINA [11, 17], BRAVO and CRUISE [31, 32], and RISE and RIDE [29], which demonstrated improvements in and maintenance of gains in VA over 1-3 years. These results demonstrate that good visual outcomes are achievable, when fixed frequent dosing regimens are properly adhered to and treatment is administered as specified.

To date, however, these results have not typically been reflected in the clinic when PRN regimens are employed, with 
- Initiate treatment with loading doses until disease is stable

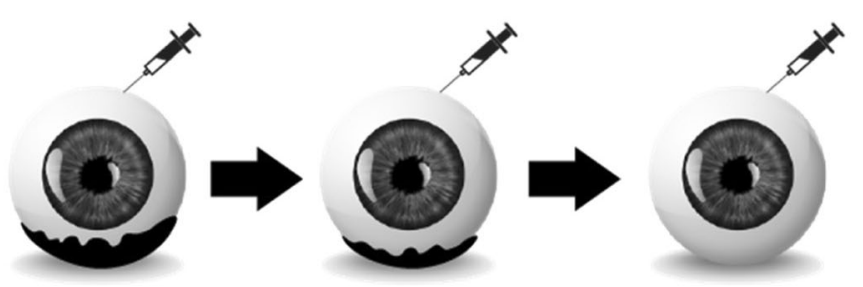

- Gradually extend treatment time between treatments until fluid recurs or VA falls, i.e. determine the "maximal fluid-free interval"

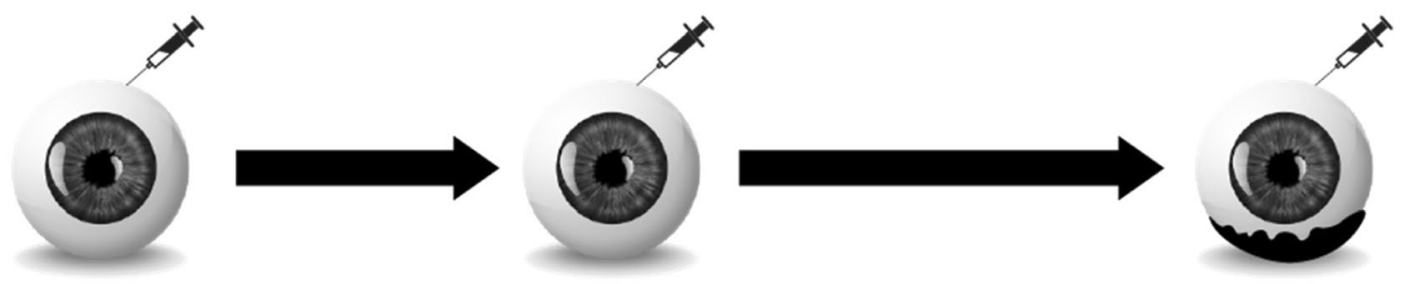

- Treat more frequently than maximal fluid-free interval to keep retina dry (i.e. treat proactively) and maintain vision

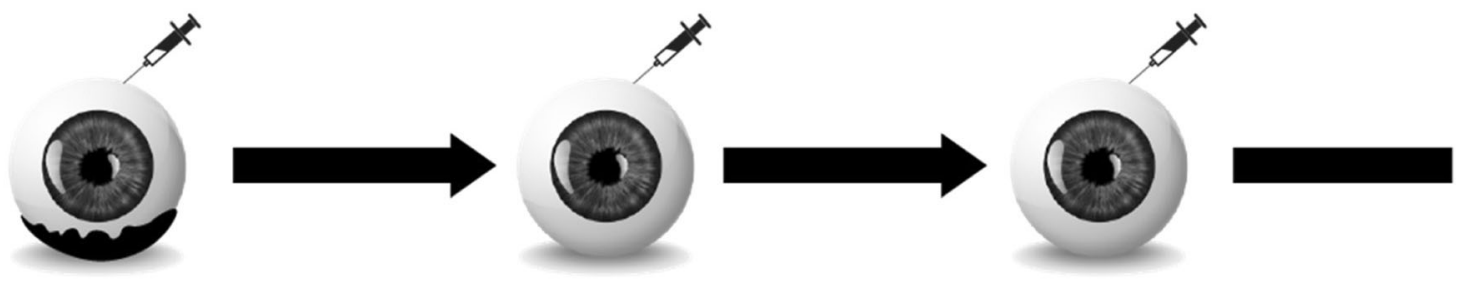

Fig. 1 Illustrative example of how a treat-and-extend regimen might be implemented in the clinic

patients experiencing mean changes of between -0.8 and +3 letters following 12 months of ranibizumab treatment [53-57].

The UK Electronic Medical Record (EMR) Users Group analyzed patient records from 12,951 eyes of 11,135 nAMD patients across the UK, and showed that there was a mean gain of only 2 ETDRS letters over the first year of treatment with ranibizumab, and that these initial gains were not maintained beyond the first year [53]. In this study, the mean number of injections administered in the first year was 5.7 , but this dropped to 3.7 in year 2 .

A more recent comprehensive review of 12 -month outcomes as reported from real-world studies with ranibizumab for the treatment of nAMD also revealed that the weighted mean change in VA from baseline to month 12 was only +1.95 ETDRS letters [68]. In AURA (a retrospective, observational study conducted in Canada, France, Germany, Ireland, Italy, the Netherlands, the UK, and Venezuela), the medical records of 2,227 patients with nAMD treated with ranibizumab were evaluated [56]. The mean improvements in VA from baseline to years 1 and 2 were 2.4 and 0.6 letters respectively; the mean number of injections administered in the first and second year were 5.0 and 2.2 respectively (see Fig. 2 for outcomes stratified by country). It seems that unless a strict schedule of monthly monitoring visits and retreatment criteria can be adhered to [49], there is a risk that disease recurrence might not be detected sufficiently early for optimal treatment outcomes.

In DME too, suboptimal outcomes in VA will normally arise due to insufficient administration of therapy, leading to under-treatment. Comparing DME trials shows that when therapy is administered via a fixed dosing regimen [46], or a strict PRN regimen with clear retreatment criteria (Protocol T) [49], then gains in VA are maximized, with optimal outcomes for the patients. However, the results from RESTORE, REVEAL, and RETAIN [30, 33, 69], which have less strict PRN treatment criteria, show less favorable VA gains and fewer injections in year 1 compared with Protocol T. Similar outcomes are also observed through a comparison of trials with ranibizumab in nAMD, where PRN regimens are generally less effective than fixed dosing regimens $[11,17,40,70$, 71].

A study by Oubraham et al. [72] highlighted the need for adequate treatment of all patients to achieve maximum gains in VA. This 12-month retrospective study investigated the efficacy of ranibizumab in nAMD patients, with therapy administered via either PRN $(n=52)$ or treat-and-extend $(n=38)$. Differences in VA gains between the two groups of patients were substantial, with those on the treat-and-extend regimen gaining a total of $10.8 \pm 8.8$ ETDRS letters vs $2.3 \pm 17.4$ ETDRS letters $(p=0.036)$ gained by patients treated according to the PRN regimen. This difference in outcomes was 
Fig. 2 Outcomes from AURA: changes in VA from baseline per country over 2 years (only countries enrolling $>400$ participants have been displayed) [55]. $L O C F$, last observation carried forward

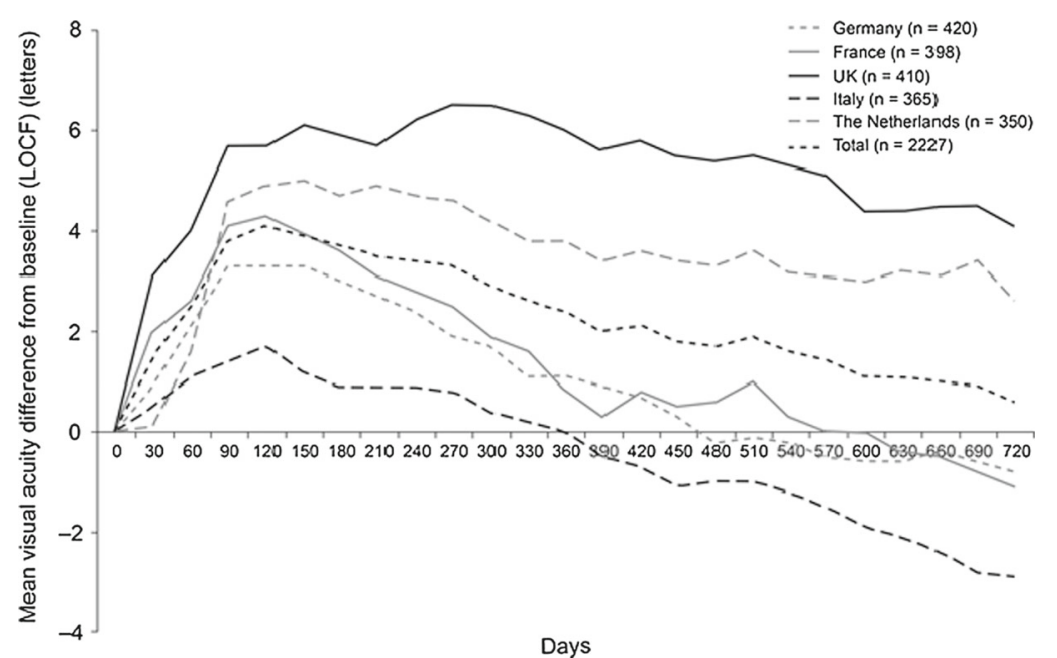

\begin{tabular}{ccc}
\hline $\begin{array}{c}\text { Countries enrolling } \\
>400 \text { participants }\end{array}$ & $\begin{array}{c}\text { Mean number of injections } \\
\text { over 2 years }\end{array}$ & $\begin{array}{c}\text { Mean number of visits } \\
\text { over 2 years }\end{array}$ \\
\hline UK & 9.0 & 18.4 \\
\hline The Netherlands & 8.7 & 12.7 \\
\hline France & 6.3 & 13.4 \\
\hline Germany & & 10.8 \\
\hline Italy & 5.6 & 12.7 \\
\hline
\end{tabular}

attributed to patients in the treat-and-extend group receiving significantly more injections than those in the PRN group (mean: $7.8 \pm 1.3$ vs $5.2 \pm 1.9 ; p<0.001$ ), despite a similar number of follow-up visits (mean: $8.5 \pm 1.1$ vs $8.8 \pm 1.5$; $p<$ $0.20)$. The group also tested the hypothesis that the difference in outcomes was driven by differences in the number of patients experiencing vision loss between the two groups; there were indeed significantly fewer patients with VA loss in the treat-and-extend group compared with the PRN group $(2.4 \%$ vs $34.6 \%$; $p=0.002$ ).

Data from the AURA trial also provide a compelling example of the importance of maintaining a sufficient injection frequency as well as the importance of close monitoring. When results from the trial were stratified by country [56] (see Fig. 2), outcomes in Italy (where patients received the fewest injections) were worst, whereas outcomes in the UK (where patients had the highest number of injections) were far better, with greater improvements in VA. However, comparison of results from Canada with the UK reveal that while a similar number of injections were administered over 2 years (a mean of 9.9 vs 9.0, respectively), there was a greater difference in the number of visits (a mean of 13.6 vs 18.4, respectively). As a result, the mean change in VA at the end of year 2 was much lower in Canada (+1.6 ETDRS letters) than in the UK (+4.1 ETDRS letters), highlighting the importance of adequate monitoring in addition to a sufficient injection frequency. It should be noted that there was a difference in baseline VA between the two countries, with values of $47.2 \pm$ 18.8 letters (Canada) and $55.0 \pm 17.8$ letters (UK).

Equally important to the concept of maximizing gains in VA is the early initiation of treatment, ideally within the first stages of disease onset. In the key intravitreal aflibercept trials in macular edema following RVO, immediate vision gains of 12-13 letters were seen after the first intravitreal aflibercept injection [67, 73, 74]. However, in COPERNICUS and GALILEO, the visual outcomes among patients initially randomized to receive 6 months of sham injections and switched to intravitreal aflibercept were inferior to those of patients who had been treated with intravitreal aflibercept from the outset [73-75]. For instance, in COPERNICUS, the mean changes in BCVA from baseline to week 100 in these two groups were +1.5 and +13.0 ETDRS letters respectively [75].

Similarly, a subgroup analysis of VIEW 1 and 2 highlighted the difficulty in trying to regain vision once it had been lost. A 
subgroup of patients in this trial were treated until stable and then only re-treated if vision deteriorated. Even if re-treated, this group never 'caught up' in terms of VA gains compared with those who were proactively treated [76, 77]. Certain subgroups of patients are also at a high risk of progressive visual loss if under-treated, e.g., approximately $50 \%$ of patients with newly diagnosed pigment epithelial detachment will experience significant visual loss ( $>3$ lines) 1 year from diagnosis [78].

Also, Lim et al. [79] investigated the effects of a delay in treatment on VA outcomes. Results from 185 eyes with nAMD showed that a delay from initial signs suggestive of $\mathrm{CNV}$ to first injection was a significant predictor $(p=0.015)$ of poorer treatment outcomes (when controlling for age, sex, and baseline VA). In addition, a delay in treatment of 21 weeks or more compared with a delay of 7 weeks had an odds ratio of $2.63(1.20,5.68)$ for worsening vision after treatment.

It is clear from these studies that waiting for further disease progression before taking therapeutic action may be detrimental to the concept of maximizing VA gains, something that the early initiation of therapy is key to. Following this, maintenance of initial gains in VA must then be considered. Declines in VA are often seen in patients exiting these clinical trials [80], as demonstrated by the 5-year outcomes from CATT [70]. In this study, head-to-head comparisons between ranibizumab and bevacizumab were conducted when the two agents were administered via fixed and PRN regimens. After 2 years, patients were released from the clinical trial protocol, and were recalled for examination at 5 years. The mean change in VA at 5 years was -3 letters from baseline, and -11 letters from 2 years. This suggests that discrepancies in visual outcomes between clinical trials and real-world practice may be explained by changes in disease management. An example of this would be a change in the frequency of injections and/or monitoring.

The SEVEN-UP trial evaluated VA 7-8 years after initiation of ranibizumab therapy in 65 nAMD patients originally treated in the ANCHOR, MARINA, and HORIZON trials. Results showed a substantial decline in baseline VA, with one-third of patients experiencing a deterioration of 15 letters or more [80]. In this study, a mean total of 6.8 injections were administered over a mean 3.4-year period. One must also consider that patients who responded poorly to treatment would most likely have exited the initial studies, leaving only those who were experiencing better outcomes to continue the longterm study.

More promising results have been observed by the National Aflibercept UK Users Group, which documented visual and anatomic outcomes in 1,840 treatment-naive eyes of patients with nAMD who were treated with intravitreal aflibercept in accordance with the VIEW protocol. By the end of year 1, a median of eight injections had been administered over a median of eight visits per patient, resulting in a mean gain of 5.1 ETDRS letters [81].
A post-hoc analysis of VIEW [77] revealed that in the second year of treatment there was a subgroup of patients (approximately 20\%) who suffered declines in vision when switching from fixed to PRN treatment. These results highlight the importance of considering the response to treatment in individual patients rather than the response observed in the whole patient population, as the latter may be driven by the best-responding patients. The evidence indicates that in order to maximize and maintain gains in VA across the patient population, regular treatment is necessary; deviations from this lead to suboptimal outcomes and a reduction in benefits for all patients, with the potential for significant vision loss in some individuals.

To summarize:

- Maximizing and maintaining gains in VA should be the aim of anti-VEGF treatment for all patients, not just those who respond well to therapy

- Early initiation of therapy and a sufficient frequency of injections are both essential for maximizing and maintaining gains in VA

\section{Decide when to treat next, rather than whether to treat now}

In any disease, allowing for reactivation or unchecked progression typically leads to adverse consequences and progression of subclinical effects. These may be small or negligible at each event, but can accumulate and have significant long-term consequences, as demonstrated in patients with severe hemophilia A [82] and multiple sclerosis [83]. It is therefore good practice to avoid such episodes and stay ahead of the disease, adopting a proactive treatment approach, in which therapy is administered to minimize the risk of disease recurrence, rather than administered in response to it. In the management of retinal diseases, each clinic visit should therefore be seen as a treatment visit and an opportunity to decide, based on the current VA and anatomic status, when therapy should be administered next. These principles are at the heart of both fixed frequent and treat-and-extend treatment regimens.

The HORIZON trial investigated the efficacy of ranibizumab administered via an investigator-led PRN regimen in AMD patients who had previously exited (and completed) the ANCHOR and MARINA trials [84] (patients were also included from the FOCUS trial, which investigated the efficacy of ranibizumab combined with verteporfin PDT for the treatment of nAMD [85]). The switch from a strict monthly ranibizumab regimen to a more relaxed PRN regimen with less frequent follow-up not only resulted in worsening and progression of AMD in patients, but also a decline in the VA gains achieved on the monthly regimen [84]. 
In terms of improvements in VA and better outcomes for the patient, current evidence indicates that treat-and-extend regimens can also result in better visual outcomes compared with PRN regimens [72, 77, 86, 87]. A study by Hatz et al., in which 146 eyes from 134 treatment-naive patients with nAMD were switched from a PRN regimen to a treat-andextend regimen, showed that greater improvements in vision were achieved following the switch in regimen [87]. After making the switch, not only did the mean BCVA (decimal) significantly improve from 0.49 at baseline to 0.55 and 0.56 at months 6 and 12 respectively (mean change in $\mathrm{BCVA}=0.06$, $p<0.001$ ), but the mean central retinal thickness (CRT) also decreased from a baseline of $355 \pm 112 \mu \mathrm{m}$ to $330 \pm 105 \mu \mathrm{m}$ and $320 \pm 103 \mu \mathrm{m}$ at months 6 and $12(p<0.001)$ respectively. In addition, the mean number of visits declined during treatand-extend. In the Oubraham et al. study previously discussed [72], nAMD patients on a treat-and-extend regimen demonstrated better outcomes in VA, with a significantly reduced risk of vision loss compared with their counterparts on the PRN regimen $(2.6 \%$ vs $34.6 \% ; p=0.002)$, despite a similar number of visits.

These studies highlight the potential limitations of a PRN regimen; there may be a greater burden of appointments compared with a treat-and-extend regimen, but improvements in VA are likely to be suboptimal without frequent monitoring and strict retreatment criteria; achieving a successful PRN regimen therefore presents a significant challenge in clinical practice.

In a more recent study, patients with nAMD were treated with intravitreal aflibercept on a treat-and-extend regimen for 6 months, following prior treatment on a fixed bimonthly regimen for the first 12 months (with treatment initiated by three monthly loading doses) [88]. BCVA improved from 60.9 letters at baseline to 68.1 letters at month $12(p<0.001)$, and to 69.6 letters at month $18(p<0.001)$.

Additional evidence for treat-and-extend has been provided by ATLAS, a trial investigating the effects of intravitreal aflibercept administered via a treat-and-extend regimen in nAMD patients [89]. Over the course of 1 year, patients received a mean of eight injections, gaining between 10 and 11 ETDRS letters. By the end of the second year, an additional mean of 5.9 injections had been administered, and improvements in VA still stood at 8-11 letters.

The knowledge that an injection will be received at every visit and the predictable timing of the next injection could have a positive effect on patient experiences. Adherence to treatment regimens is crucial in clinical practice; betterinformed patients not only experience reduced treatment burden but in turn have a positive impact on clinic operation. Knowing the timing of the next injection may also serve to maximize access to treatment in health systems where approval is required prior to the next injection, by allowing the physician more time to submit the necessary paperwork.
To summarize:

- The success of anti-VEGF treatment depends not only on the treatment of active disease, but also on the prevention of disease recurrence and/or worsening

- Planning the next anti-VEGF treatment helps to minimize the possibility of delays in treatment, allows time for treatment approval to be obtained if needed, and facilitates clinic management. Patients may also benefit from being able to anticipate and plan for their next injection in good time

- A proactive approach, such as treat-and-extend, allows physicians to stay ahead of the disease and, by minimizing the need for intervening visits, helps to ease the burden on clinics and patients

\section{Titrate the treatment intervals to match patients' needs}

The duration of VEGF-A suppression differs between patients and treatment. For example, intraocular measurements have revealed that VEGF-A suppression times in nAMD patients treated with ranibizumab can vary between patients from 26 to 69 days (Fig. 3) [90]. While different between patients, the suppression times were stable for each individual over a period of 3 years, with a mean suppression time of 36.4 days. This intra-individual stability has also been demonstrated in experiments investigating the suppression of VEGF by intravitreal aflibercept [91], which has been shown to suppress VEGF in the eyes of patients with nAMD for a mean of 71 days [91]. Although VEGF suppression times differ between patients, the consequences of this suppression are unclear. There are limited data available that link intraocular VEGF levels with outcomes; however, a recent study demonstrated that VEGF suppression time correlated with reductions in central retinal volume following treatment with either ranibizumab or aflibercept [92].

Support for the biological difference between patients may also be found in clinical trial data. Post-hoc analyses of VIEW demonstrated that in the second year of treatment, approximately $48 \%$ of patients treated with intravitreal aflibercept required three or fewer injections between weeks 52 to 96 [77]. Similarly, in the second year of treatment in ATLAS, $82 \%$ of patients with nAMD had a treatment interval of 8 weeks or more, while $41 \%$ of patients had a treatment interval of 12 weeks or more [89] (see Fig. 4). The LUCAS trial also demonstrated differences in the mean number of treatments and intervals between nAMD patients treated with bevacizumab or ranibizumab administered via a treat-andextend regimen, over the course of 2 years [93]. The percentage of patients achieving a treatment interval of 8 weeks or more was approximately $34 \%$ in the bevacizumab group and $47 \%$ in the ranibizumab group, while the percentage treated 


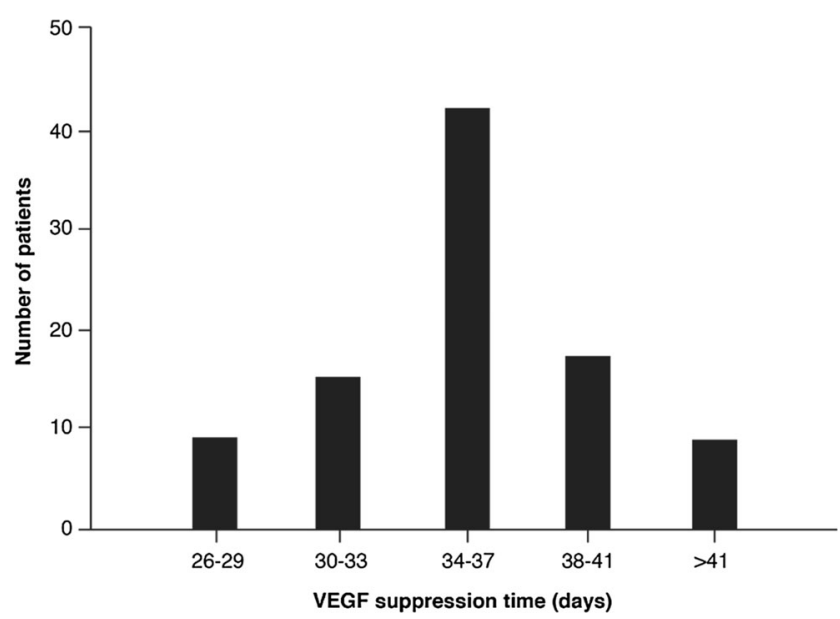

Fig. 3 Distribution of individual VEGF suppression times of patients with nAMD treated with ranibizumab, as reported in Muether et al. [89]

every 12 weeks was approximately $20 \%$ in the bevacizumab group and $33 \%$ in the ranibizumab group.

Mantel et al. [94] also investigated the predictability of the need for retreatment in nAMD patients. Treatment was initiated with three monthly loading doses of ranibizumab, after which patients were monitored on a weekly basis, with stepwise increases to every 2 weeks and then to monthly after each injection. Retreatment occurred on an as-needed basis. Results showed that the first interval between treatments after the loading phase was a good predictor of the following treatment intervals, and that there was high intra-individual predictability of retreatment need for patients with nAMD. This supports the notion of individualized treatment plans for patients, with a minimum number of injections and visits but maintained suppression of disease activity. Separate investigations support the idea that intra-individual stability of VEGF

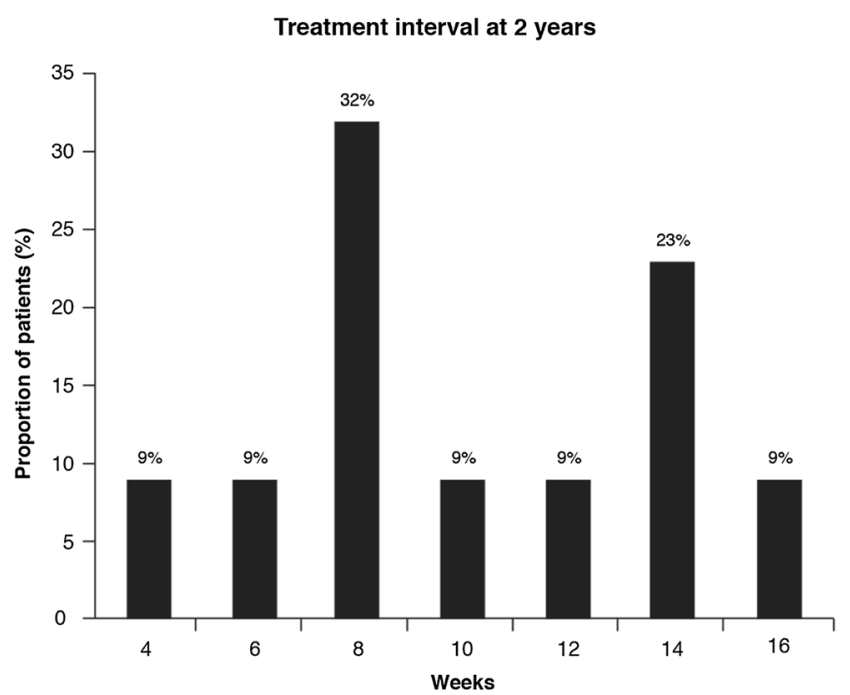

Fig. 4 Distribution of treatment intervals in patients receiving intravitreal aflibercept in the ATLAS study [89] suppression is not exclusive to patients with nAMD, having also been observed in patients with DME [95].

Similarly, subgroup analysis of patients treated with intravitreal aflibercept in COPERNICUS and GALILEO revealed that after the initial 6-month period, some patients with macular edema following CRVO required fewer than three injections in the following 6 months to maintain vision [96]. Whether additional therapy would have led to improvements in VA is unknown.

A more individualized approach to treatment may encourage patients to adhere to treatment schedules by specifically tailoring the treatment regimen to their needs. Extension of intervals between treatments also sends a message that treatment is 'working', which may lift patients' spirits and lead to renewed enthusiasm for treatment. This is in contrast with a PRN regimen, in which the marker for retreatment is anatomic recurrence or a decline in vision, which could be perceived as a 'failure' of treatment, thus negatively affecting patient psychology.

Physicians should be aware of the risks of PRN with monthly monitoring, which leaves the patient open to a potential risk of disease reactivation (see Fig. 5). To avoid this, an understanding of the intraocular durability of the various antiVEGF agents is needed.

Studies in AMD, DME, and RVO have shown intravitreal aflibercept $2 \mathrm{q} 8$ to be equally as efficacious as intravitreal aflibercept $2 \mathrm{q} 4[40,46,97]$ and, in nAMD, also equivalent to ranibizumab $0.5 \mathrm{q} 4$ [40]. In a study by Pfau et al. [98], 13 patients with macular edema following CRVO requiring treatment with bevacizumab or ranibizumab at least every 6 weeks were switched to therapy with intravitreal aflibercept, administered via a treat-and-extend regimen. Twelve months after switching, the mean ETDRS letter score increased from 66.51 at baseline to $76.54(p=0.021)$, and the mean CRT decreased by $195.84 \mu \mathrm{m}$. The mean injection interval also increased by 0.51 months $(p=0.023)$ and the relapse-free interval by 3.02 weeks $(p=0.003)$.

The NEWTON trial [99] (which investigated the effect of switching from bevacizumab or ranibizumab to intravitreal aflibercept in patients with macular edema following CRVO) demonstrated that upon switching, the treatment interval could be extended from a mean 5-6 weeks to 8 weeks, with a 7.0letter improvement in VA $(p=0.01)$ and a decrease in retinal thickness by $145 \mu \mathrm{m}(p<0.01)$. The average central macular edema-free interval also increased from 37 to 65 days $(p<$ $0.0001)$.

This again links in with the idea of patient individuality and a difference in needs. By titrating the treatment interval to suit the patient's needs and adjusting the treatment if necessary, physicians could achieve optimal outcomes for each patient through careful extension of the intervals between treatments, and aim to alleviate some of the burden on patients and clinics. 


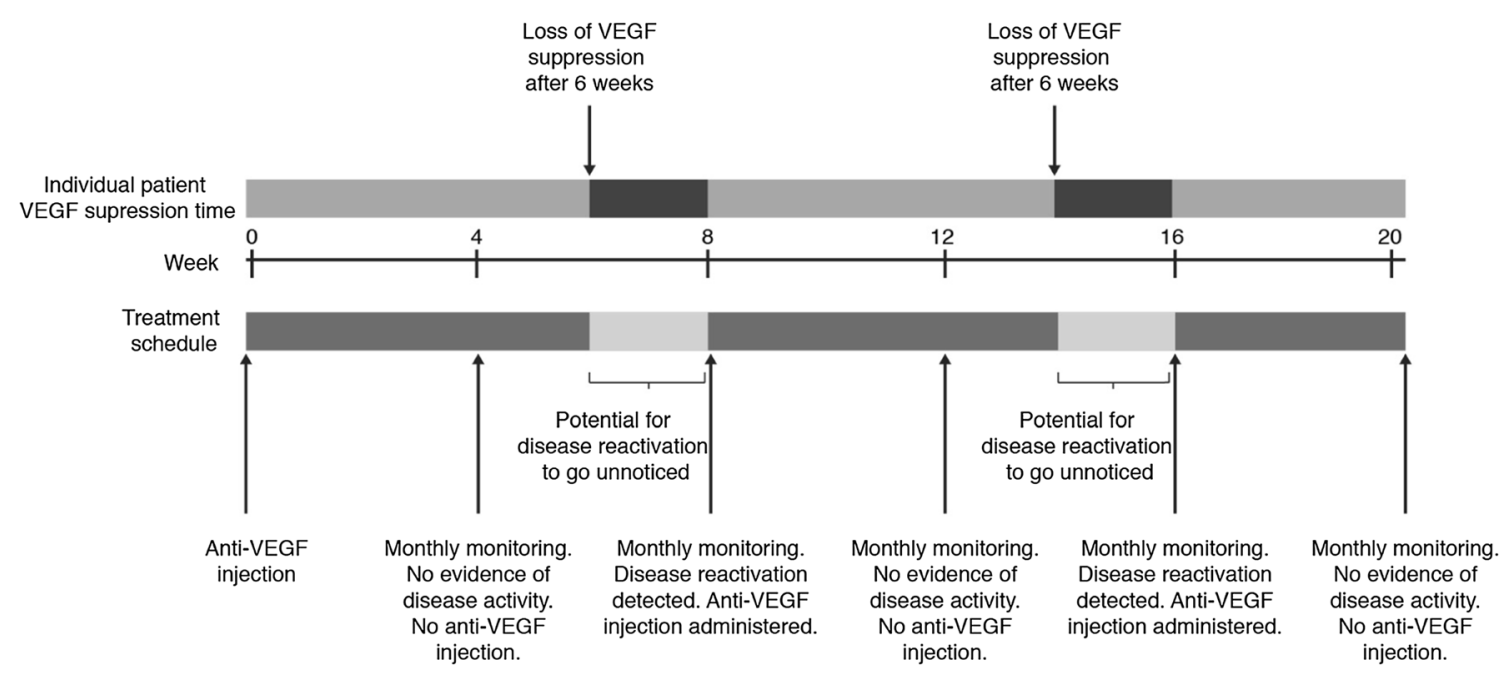

Fig. 5 PRN treatment with monthly monitoring leaves patients at risk of disease resurgence

To summarize:

The duration of VEGF suppression varies between patients and differs between anti-VEGF agents

Anti-VEGF agents with greater duration of action might allow for longer extension of treatment intervals than for those with shorter durabilities

Customization of the treatment interval to the individual patient removes the need for interim monitoring, while achieving optimal outcomes for the patient

\section{Treat at each monitoring visit}

According to this concept, patients should be treated at every monitoring visit, meaning that no opportunity is missed to treat the disease. This simple change in practice has the potential to have a positive impact on the management of the disease; clinic flow would be improved by reducing the number of appointments per patient, and the possibility of unidentified disease recurrence would be minimized by the crucial elimination of any delay between assessment and treatment. Such delays (common in PRN regimens) often result in a change or worsening of disease status. Using each visit to collect the information required to enable treatment at the following visit should also help to ensure that treatment is delivered on time.

In a retrospective study by Oubraham et al. [72], the poor VA outcomes achieved by patients on a PRN regimen were attributed to a delay between identification and treatment of recurrences, which might potentially lead to irreversible foveal damage [100]. Such delays were avoided in the treat-and-extend arm of the study, in which the VA outcomes achieved were superior to those achieved by patients receiving treatment via the PRN regimen (particularly with respect to vision loss).

With consideration of the practicalities of managing multiple clinic appointments, scheduling one appointment for both treatment and monitoring should make it easier for patients to plan and manage travel to and from clinics, especially for patients who have long distances to cover or who need to be accompanied [61]. Furthermore, such a proactive approach, and the certainty that each clinic visit will involve an injection, may alleviate the stress experienced by some patients.

By using each clinic visit as an opportunity to monitor and treat, no chance is missed to stay ahead of the disease, and the physician will be able to make the most out of every interaction with the patient. This is important, as the literature shows that a delay in treatment or in treating recurrences of the disease can lead to suboptimal outcomes for the patient $[72,76$, 77, 84].

While continued monitoring and treatment is part of the treat-and-extend strategy, no precise exit strategy has been defined for this approach as yet. The durability of anti-VEGF treatment has been demonstrated by longterm studies of up to 8 years [80], but further studies will be needed to determine whether treatment can be tapered and stopped completely following a treat-andextend regimen.

To summarize:

- Monitoring and treating within the same appointment helps to eliminate the possibility of disease resurgence that can occur between separate monitoring and treatment appointments

- The number of appointments per patient will be reduced, helping to ease clinic flow and patient burden, and also reducing the stress experienced by the patient 


\section{Discussion and summary}

The ideal treatment regimen should be effective, proactive, individualized, and convenient. In summary, the following four principles are proposed as fundamental to an ideal treatment regimen for the treatment of chronic retinal disorders:

1. Maximize and maintain VA benefits for all patients

2. Decide when to treat next, rather than whether to treat now

3. Titrate the treatment intervals to match patients' needs

4. Treat at each monitoring visit.

Together, the four principles point toward the implementation of a predictable, proactive and manageable treatment regimen, with consideration of individual patient needs and minimization of delays in treatment. If all four principles are implemented in practice, they are anticipated to lead to benefits for both patient and physician, with better organization of clinics, improved utilization of resources, and increased clinic capacity. Adopting a more personalized approach and reduced treatment burden may also lead to improvements in patient compliance.

It is hoped that implementation of a proactive regimen in intravitreal clinics will lead to further development and better understanding of appropriate treatment regimens, including treat-and-extend. There are few comparative studies that demonstrate the benefit of treat-and-extend, but the evidence base is growing, with further studies ongoing to fully characterize the impact of this approach.

While there is evidence for the benefits of treat-and-extend in nAMD (and RVO), DME is less clear, with treatment regimens in this disease area continuously evolving as more data come to light. The Protocol T data [49] highlight the effectiveness of aggressive PRN, in which the default position is to treat unless specific criteria are fulfilled. This is contrary to other PRN approaches such as in CATT [70], where treatment is withheld until certain criteria are met. It could thus be said that this remains a proactive treatment approach.

Little is also known about patients' quality of life with treat-and-extend and PRN regimens, and discussions are needed on the obstacles that may be faced when integrating treat-and-extend regimens into existing clinical practice. There should also be further discussion of defining response to treatment, and when and how to stop treatment.

It is important to note that as an 'ideal' treatment regimen, costs of treatment (including country-specific financial drivers), have not been considered. These issues will need to be addressed on a local level, by taking the principles of an ideal regimen and holding discussions on a national basis on how best to implement them.

To date, it has proven challenging to translate the excellent outcomes seen in clinical studies into real-world practice. The
Vision Academy, by recommending these four principles, anticipates that patient management can be improved, the burden on the clinic can be reduced, and outcomes for each patient can be optimized, with avoidable vision loss minimized.

Acknowledgements The authors take full responsibility for the scope, direction and content of the manuscript and have approved the submitted manuscript. Medical writing assistance was provided by Porterhouse Medical Ltd and was funded by Bayer.

\section{Compliance with ethical standards}

Funding Bayer provided financial support in the form of medical writing funding to Porterhouse Medical Ltd.

The sponsor had no role in the design or conduct of this research.

Conflicts of interest All authors certify that they have no affiliations with or involvement in any organization or entity with any financial interest (such as honoraria; educational grants; participation in speakers' bureaus; membership, employment, consultancies, stock ownership, or other equity interest; and expert testimony or patent-licensing arrangements), or non-financial interest (such as personal or professional relationships, affiliations, knowledge, or beliefs) in the subject matter or materials discussed in this manuscript.

Bora Eldem: Allergan, Bausch \& Lomb, Bayer, Novartis, Opthotec, Roche, Santen, Servier, Thea.

Alex Hunyor: Allergan, Bayer, Novartis.

Antonia Joussen: None.

Adrian Koh: Bayer.

Jean-François Korobelnik: Bayer, Novartis, Roche.

Paolo Lanzetta: Alcon, Alimera, Allergan, Bayer, Genentech, Lupin, Lutronic, Novartis, Roche,

Anat Loewenstein: Alcon, Allergan, Bayer, Notal Vision, Novartis.

Studies with human participants This article does not contain any studies with human participants performed by any of the authors.

\section{Appendix: Vision Academy Steering Committee}

The Vision Academy Steering Committee is a group of ophthalmologists from across the globe, convened by Bayer to share best practice, knowledge, and lead the wider community in the drive toward optimized patient care. The Steering Committee of the Vision Academy comprises the following members:

Bora Eldem, Hacettepe University, Turkey

Alex Hunyor, University of Sydney, Australia

Antonia Joussen, Charité - University Medicine Berlin, Germany

Adrian Koh, Eye \& Retina Surgeons, Camden Medical Centre, Singapore

Jean-François Korobelnik, University Hospital of Bordeaux, France

Paolo Lanzetta, University of Udine, Italy

Anat Loewenstein, Tel Aviv Sourasky Medical Center, Israel

Monica Lövestam-Adrian, Lund University Hospital, Sweden 
Rafael Navarro, Institute of Ocular Microsurgery, Spain

Márcio Nehemy, Federal University of Minas Gerais, Brazil

Annabelle A. Okada, Kyorin University School of Medicine, Japan

Ian Pearce, Royal Liverpool University Hospital, United Kingdom

Francisco Rodríguez, Fundación Oftalmológica Nacional, Colombia

Sebastian Wolf, Inselspital, Switzerland

David Wong, University of Toronto, Canada

Open Access This article is distributed under the terms of the Creative Commons Attribution 4.0 International License (http:// creativecommons.org/licenses/by/4.0/), which permits unrestricted use, distribution, and reproduction in any medium, provided you give appropriate credit to the original author(s) and the source, provide a link to the Creative Commons license, and indicate if changes were made.

\section{References}

1. Freund KB, Korobelnik J-F, Devenyi R, Framme C, Galic J, Herbert E, Hoerauf H, Lanzetta P, Michels S, Mitchell P, Monés J, Regillo C, Tadayoni R, Talks J, Wolf S (2015) Treat-and-extend regimens with anti-VEGF agents in retinal diseases: a literature review and consensus recommendations. Retina 35:1489-1506

2. Ishikawa M, Jin D, Sawada Y, Abe S, Yoshitomi T (2015) Future therapies of wet age-related macular degeneration. J Ophthalmol 2015:138070

3. Rogers S, McIntosh RL, Cheung N, Lim L, Wang JJ, Mitchell P, Kowalski JW, Nguyen H, Wong TY, International Eye Disease Consortium (2010) The prevalence of retinal vein occlusion: pooled data from population studies from the United States, Europe, Asia, and Australia. Ophthalmology 117:313-319

4. Wolf S (2014) Diabetic macular oedema and the importance of vascular endothelial growth factor therapies in its treatment. Eur Ophthalmic Rev 8:53-60

5. Schmidt-Erfurth U, Waldstein SM, Deak GG, Kundi M, Simader C (2015) Pigment epithelial detachment followed by retinal cystoid degeneration leads to vision loss in treatment of neovascular age-related macular degeneration. Ophthalmology 122:822-832

6. Wong WL, Su X, Li X, Cheung CM, Klein R, Cheng CY, Wong TY (2014) Global prevalence of age-related macular degeneration and disease burden projection for 2020 and 2040: a systematic review and meta-analysis. Lancet Glob Health 2:e106-e116

7. Klein R, Knudtson MD, Lee KE, Gangnon R, Klein BE (2009) The Wisconsin Epidemiologic Study of Diabetic Retinopathy XXIII: the twenty-five-year incidence of macular edema in persons with type 1 diabetes. Ophthalmology 116:497-503

8. Rein DB, Zhang P, Wirth KE, Lee PP, Hoerger TJ, McCall N, Klein R, Tielsch JM, Vijan S, Saaddine J (2006) The economic burden of major adult visual disorders in the United States. Arch Ophthalmol 124:1754-1760

9. Boyer DS, Hopkins JJ, Sorof J, Ehrlich JS (2013) Anti-vascular endothelial growth factor therapy for diabetic macular edema. Ther Adv Endocrinol Metab 4:151-169
10. Brown DM, Kaiser PK, Michels M, Soubrane G, Heier JS, Kim RY, Sy JP, Schneider S, ANCHOR Study Group (2006) Ranibizumab versus verteporfin for neovascular age-related macular degeneration. N Engl J Med 355:1432-1444

11. Brown DM, Michels M, Kaiser PK, Heier JS, Sy JP, Ianchulev T, ANCHOR Study Group (2009) Ranibizumab versus verteporfin photodynamic therapy for neovascular age-related macular degeneration: two-year results of the ANCHOR study. Ophthalmology 116:57-65

12. Ford JA, Lois N, Royle P, Clar C, Shyangdan D, Waugh N (2013) Current treatments in diabetic macular oedema: systematic review and meta-analysis. BMJ Open 3:e002269

13. Freund KB, Mrejen S, Gallego-Pinazo R (2013) An update on the pharmacotherapy of neovascular age-related macular degeneration. Expert Opin Pharmacother 14:1017-1028

14. Jain A, Varshney N, Smith C (2013) The evolving treatment options for diabetic macular edema. Int J Inflam 2013:689276

15. Kaiser PK, Boyer DS, Cruess AF, Slakter JS, Pilz S, Weisberger A, DENALI Study Group (2012) Verteporfin plus ranibizumab for choroidal neovascularization in age-related macular degeneration: twelve-month results of the DENALI study. Ophthalmology 119: $1001-1010$

16. Lövestam-Adrian M, Agardh E (2000) Photocoagulation of diabetic macular oedema - complications and visual outcome. Acta Ophthalmol Scand 78:667-671

17. Rosenfeld PJ, Brown DM, Heier JS, Boyer DS, Kaiser PK, Chung CY, Kim RY, MARINA Study Group (2006) Ranibizumab for neovascular age-related macular degeneration. N Engl J Med 355:1419-1431

18. Ishida S, Usui T, Yamashiro K, Kaji Y, Ahmed E, Carrasquillo KG, Amano S, Hida T, Oguchi Y, Adamis AP (2003) VEGF164 is proinflammatory in the diabetic retina. Invest Ophthalmol Vis Sci 44:2155-2162

19. Ishida S, Usui T, Yamashiro K, Kaji Y, Amano S, Ogura Y, Hida T, Oguchi Y, Ambati J, Miller JW, Gragoudas ES, Ng YS, D'Amore PA, Shima DT, Adamis AP (2003) VEGF164-mediated inflammation is required for pathological, but not physiological, ischemia-induced retinal neovascularization. J Exp Med 198: 483-489

20. Usui T, Ishida S, Yamashiro K, Kaji Y, Poulaki V, Moore J, Moore T, Amano S, Horikawa Y, Dartt D, Golding M, Shima DT, Adamis AP (2004) VEGF164(165) as the pathological isoform: differential leukocyte and endothelial responses through VEGFR1 and VEGFR2. Invest Ophthalmol Vis Sci 45:368-374

21. Ahmadi MA, Lim JI (2008) Pharmacotherapy of age-related macular degeneration. Expert Opin Pharmacother 9:3045-3052

22. Rosenfeld PJ, Fung AE, Puliafito CA (2005) Optical coherence tomography findings after an intravitreal injection of bevacizumab (Avastin) for macular edema from central retinal vein occlusion. Ophthalmic Surg Lasers Imaging 36:336-339

23. Moja L, Lucenteforte E, Kwag KH, Bertele V, Campomori A, Chakravarthy U, D'Amico R, Dickersin K, Kodjikian L, Lindsley K, Loke Y, Maguire M, Martin DF, Mugelli A, Muhlbauer B, Puntmann I, Reeves B, Rogers C, Schmucker C, Subramanian ML, Virgili G (2014) Systemic safety of bevacizumab versus ranibizumab for neovascular age-related macular degeneration. Cochrane Database Syst Rev:CD011230

24. Tah V, Orlans HO, Hyer J, Casswell E, Din N, Sri Shanmuganathan V, Ramskold L, Pasu S (2015) Anti-VEGF therapy and the retina: an update. J Ophthalmol 2015:627674

25. Nguyen QD, Brown DM, Marcus DM, Boyer DS, Patel S, Feiner L, Gibson A, Sy J, Rundle AC, Hopkins JJ, Rubio RG, Ehrlich JS, RISE and RIDE Research Group (2012) Ranibizumab for diabetic macular edema: results from 2 phase III randomized trials: RISE and RIDE. Ophthalmology 119:789-801 
26. Varma R, Bressler NM, Suñer I, Lee P, Dolan CM, Ward J, Colman S, Rubio RG, BRAVO and CRUISE Study Groups (2012) Improved vision-related function after ranibizumab for macular edema after retinal vein occlusion: results from the BRAVO and CRUISE trials. Ophthalmology 119:2108-2118

27. Tufail A, Narendran N, Patel PJ, Sivaprasad S, Amoaku W, Browning AC, Osoba O, Gale R, George S, Lotery AJ, Majid M, McKibbin M, Menon G, Andrews C, Brittain C, Osborne A, Yang Y (2013) Ranibizumab in myopic choroidal neovascularization: the 12-month results from the REPAIR study. Ophthalmology 120:1944-1945

28. Wolf S, Balciuniene VJ, Laganovska G, Menchini U, OhnoMatsui K, Sharma T, Wong TY, Silva R, Pilz S, Gekkieva M, RADIANCE Study Group (2014) RADIANCE: a randomized controlled study of ranibizumab in patients with choroidal neovascularization secondary to pathologic myopia. Ophthalmology 121:682-692

29. Brown DM, Nguyen QD, Marcus DM, Boyer DS, Patel S, Feiner L, Schlottmann PG, Rundle AC, Zhang J, Rubio RG, Adamis AP, Ehrlich JS, Hopkins JJ, RIDE and RISE Research Group (2013) Long-term outcomes of ranibizumab therapy for diabetic macular edema: the 36-month results from two phase III trials: RISE and RIDE. Ophthalmology 120:2013-2022

30. Ishibashi T, Li X, Koh A, Lai TY, Lee FL, Lee WK, Ma Z, Ohji M, Tan N, Cha SB, Shamsazar J, Yau CL, REVEAL Study Group (2015) The REVEAL study: ranibizumab monotherapy or combined with laser versus laser monotherapy in Asian patients with diabetic macular edema. Ophthalmology 122:1402-1415

31. Brown DM, Campochiaro PA, Bhisitkul RB, Ho AC, Gray S, Saroj N, Adamis AP, Rubio RG, Murahashi WY (2011) Sustained benefits from ranibizumab for macular edema following branch retinal vein occlusion: 12-month outcomes of a phase III study. Ophthalmology 118:1594-1602

32. Campochiaro PA, Brown DM, Awh CC, Lee SY, Gray S, Saroj N, Murahashi WY, Rubio RG (2011) Sustained benefits from ranibizumab for macular edema following central retinal vein occlusion: twelve-month outcomes of a phase III study. Ophthalmology 118:2041-2049

33. Mitchell P, Bandello F, Schmidt-Erfurth U, Lang GE, Massin P, Schlingemann RO, Sutter F, Simader C, Burian G, Gerstner O, Weichselberger A, RESTORE study group (2011) The RESTORE study: ranibizumab monotherapy or combined with laser versus laser monotherapy for diabetic macular edema. Ophthalmology 118:615-625

34. Schmidt-Erfurth U, Lang GE, Holz FG, Schlingemann RO, Lanzetta P, Massin P, Gerstner O, Bouazza AS, Shen H, Osborne A, Mitchell P, RESTORE Extension Study Group (2014) Three-year outcomes of individualized ranibizumab treatment in patients with diabetic macular edema: the RESTORE extension study. Ophthalmology 121:1045-1053

35. Holash J, Davis S, Papadopoulos N, Croll SD, Ho L, Russell M, Boland P, Leidich R, Hylton D, Burova E, Ioffe E, Huang T, Radziejewski C, Bailey K, Fandl JP, Daly T, Wiegand SJ, Yancopoulos GD, Rudge JS (2002) VEGF-Trap: a VEGF blocker with potent antitumor effects. Proc Natl Acad Sci U S A 99: 11393-11398

36. Rudge JS, Holash J, Hylton D, Russell M, Jiang S, Leidich R, Papadopoulos N, Pyles EA, Torri A, Wiegand SJ, Thurston G, Stahl N, Yancopoulos GD (2007) VEGF Trap complex formation measures production rates of VEGF, providing a biomarker for predicting efficacious angiogenic blockade. Proc Natl Acad Sci U S A 104:18363-18370

37. Stewart MW (2012) Aflibercept (VEGF Trap-eye): the newest anti-VEGF drug. Br J Ophthalmol 96:1157-1158
38. Kanda A, Noda K, Saito W, Ishida S (2015) Aflibercept traps galectin-1, an angiogenic factor associated with diabetic retinopathy. Sci Rep 5:17946

39. Lanzetta P, Mitchell P, Wolf S, Veritti D (2013) Different antivascular endothelial growth factor treatments and regimens and their outcomes in neovascular age-related macular degeneration: a literature review. Br J Ophthalmol 97:1497-1507

40. Schmidt-Erfurth U, Kaiser PK, Korobelnik J-F, Brown DM, Chong V, Nguyen QD, Ho AC, Ogura Y, Simader C, Jaffe GJ, Slakter JS, Yancopoulos GD, Stahl N, Vitti R, Berliner AJ, Soo Y, Anderesi M, Sowade O, Zeitz O, Norenberg C, Sandbrink R, Heier JS (2014) Intravitreal aflibercept injection for neovascular age-related macular degeneration: ninety-six-week results of the VIEW studies. Ophthalmology 121:193-201

41. Boyer D, Heier J, Brown DM, Clark WL, Vitti R, Berliner AJ, Groetzbach G, Zeitz O, Sandbrink R, Zhu X, Beckmann K, Haller JA (2012) Vascular endothelial growth factor Trap-Eye for macular edema secondary to central retinal vein occlusion: six-month results of the phase 3 COPERNICUS study. Ophthalmology 119: 1024-1032

42. Heier JS, Clark WL, Boyer DS, Brown DM, Vitti R, Berliner AJ, Kazmi H, Ma Y, Stemper B, Zeitz O, Sandbrink R, Haller JA (2014) Intravitreal aflibercept injection for macular edema due to central retinal vein occlusion: two-year results from the COPERNICUS study. Ophthalmology 121:1414-1420

43. Ogura Y, Roider J, Korobelnik J-F, Holz FG, Simader C, SchmidtErfurth U, Vitti R, Berliner AJ, Hiemeyer F, Stemper B, Zeitz O, Sandbrink R, GALILEO Study Group (2014) Intravitreal aflibercept for macular edema secondary to central retinal vein occlusion: 18-month results of the phase 3 GALILEO study. Am J Ophthalmol 158:1032-1038

44. Sivaprasad S, Asmus F, VIBRANT Study Investigators (2015) Intravitreal aflibercept for macular edema due to branch retinal vein occlusion. Presented at the 6th World Congress on Controversies in Ophthalmology; Sorrento, Italy, March 26-29, 2015

45. Ikuno Y, Ohno-Matsui K, Wong TY, Korobelnik J-F, Vitti R, Li T, Stemper B, Asmus F, Zeitz O, Ishibashi T, Investigators MYRROR (2015) Intravitreal aflibercept injection in patients with myopic choroidal neovascularization: the MYRROR study. Ophthalmology 122:1220-1227

46. Brown DM, Schmidt-Erfurth U, Do DV, Holz FG, Boyer DS, Midena E, Heier JS, Terasaki H, Kaiser PK, Marcus DM, Nguyen QD, Jaffe GJ, Slakter JS, Simader C, Soo Y, Schmelter T, Yancopoulos GD, Stahl N, Vitti R, Berliner AJ, Zeitz O, Metzig C, Korobelnik J-F (2015) Intravitreal aflibercept for diabetic macular edema: 100-week results from the VISTA and VIVID studies. Ophthalmology 122:2044-2052

47. Korobelnik J-F, Do DV, Schmidt-Erfurth U, Boyer DS, Holz FG, Heier JS, Midena E, Kaiser PK, Terasaki H, Marcus DM, Nguyen QD, Jaffe GJ, Slakter JS, Simader C, Soo Y, Schmelter T, Yancopoulos GD, Stahl N, Vitti R, Berliner AJ, Zeitz O, Metzig C, Brown DM (2014) Intravitreal aflibercept for diabetic macular edema. Ophthalmology 121:2247-2254

48. Wells JA, Glassman AR, Ayala AR, Jampol LM, Bressler NM, Bressler SB, Brucker AJ, Ferris FL, Hampton GR, Jhaveri C, Melia M, Beck RW, Diabetic Retinopathy Clinical Research Network (2016) Aflibercept, bevacizumab, or ranibizumab for diabetic macular edema: two-year results from a comparative effectiveness randomized clinical trial. Ophthalmology 123:13511359

49. Diabetic Retinopathy Clinical Research Network, Wells JA, Glassman AR, Ayala AR, Jampol LM, Aiello LP, Antoszyk AN, Arnold-Bush B, Baker CW, Bressler NM, Browning DJ, Elman MJ, Ferris FL, Friedman SM, Melia M, Pieramici DJ, Sun JK, 
Beck RW (2015) Aflibercept, bevacizumab, or ranibizumab for diabetic macular edema. N Engl J Med 372:1193-1203

50. European Medicines Agency (2015) EYLEA - EPAR summary for the public, http://www.ema.europa.eu/ema/index.jsp?curl= pages/medicines/human/medicines/002392/human_med 001598.jsp\& mid $=$ WC0b01 ac058001d124 Last accessed December 28th, 2016

51. European Medicines Agency (2016) Lucentis - EPAR summary for the public, http://www.ema.europa.eu/ema/index.jsp?curl= pages/medicines/human/medicines/000715/human_med 000890.jsp\&mid=WC0b01 ac058001d124 Last accessed December 28th, 2016

52. Schmidt-Erfurth U, Eldem B, Guymer R, Korobelnik J-F, Schlingemann RO, Axer-Siegel R, Wiedemann P, Simader C, Gekkieva M, Weichselberger A, EXCITE Study Group (2011) Efficacy and safety of monthly versus quarterly ranibizumab treatment in neovascular age-related macular degeneration: the EXCITE study. Ophthalmology 118:831-839

53. Writing Committee for the UK Age-Related Macular Degeneration EMR Users Group (2014) The neovascular agerelated macular degeneration database: multicenter study of 92 976 ranibizumab injections: report 1: visual acuity. Ophthalmology 121:1092-1101

54. Cohen SY, Mimoun G, Oubraham H, Zourdani A, Malbrel C, Queré S, Schneider V, LUMIERE Study Group (2013) Changes in visual acuity in patients with wet age-related macular degeneration treated with intravitreal ranibizumab in daily clinical practice: the LUMIERE study. Retina 33:474-481

55. Holz FG, Bandello F, Gillies M, Mitchell P, Osborne A, Sheidow T, Souied E, Figueroa MS, LUMINOUS Steering Committee (2013) Safety of ranibizumab in routine clinical practice: 1-year retrospective pooled analysis of four European neovascular AMD registries within the LUMINOUS programme. Br J Ophthalmol 97:1161-1167

56. Holz FG, Tadayoni R, Beatty S, Berger A, Cereda MG, Cortez R, Hoyng CB, Hykin P, Staurenghi G, Heldner S, Bogumil T, Heah T, Sivaprasad S (2015) Multi-country real-life experience of antivascular endothelial growth factor therapy for wet age-related macular degeneration. Br J Ophthalmol 99:220-226

57. Zhu M, Chew JK, Broadhead GK, Luo K, Joachim N, Hong T, Syed A, Chang AA (2015) Intravitreal ranibizumab for neovascular age-related macular degeneration in clinical practice: five-year treatment outcomes. Graefes Arch Clin Exp Ophthalmol 253:1217-1225

58. Holz FG, Amoaku W, Donate J, Guymer RH, Kellner U, Schlingemann RO, Weichselberger A, Staurenghi G, SUSTAIN Study Group (2011) Safety and efficacy of a flexible dosing regimen of ranibizumab in neovascular age-related macular degeneration: the SUSTAIN study. Ophthalmology 118:663-671

59. Lalwani GA, Rosenfeld PJ, Fung AE, Dubovy SR, Michels S, Feuer W, Davis JL, Flynn HW Jr, Esquiabro M (2009) A variable-dosing regimen with intravitreal ranibizumab for neovascular age-related macular degeneration: year 2 of the PrONTO Study. Am J Ophthalmol 148:43-58

60. Engelbert M, Zweifel SA, Freund KB (2009) "Treat and extend" dosing of intravitreal antivascular endothelial growth factor therapy for type 3 neovascularization/retinal angiomatous proliferation. Retina 29:1424-1431

61. Droege KM, Muether PS, Hermann MM, Caramoy A, Viebahn U, Kirchhof B, Fauser S (2013) Adherence to ranibizumab treatment for neovascular age-related macular degeneration in real life. Graefes Arch Clin Exp Ophthalmol 251:1281-1284

62. Barzey V, Muston D, Gerlinger C, Lloyd A (2015) Relationship between visual acuity and ability to perform activities in patients with diabetic macular edema. Presented at the 15th ESASO Retina Academy 2015; Barcelona, Spain, October 22-24, 2015
63. Bressler NM, Varma R, Mitchell P, Suñer IJ, Dolan C, Ward J, Ferreira A, Ehrlich JS, Turpcu A (2016) Effect of ranibizumab on the decision to drive and vision function relevant to driving in patients with diabetic macular edema: report from RESTORE, RIDE, and RISE trials. JAMA Ophthalmol 134:160-166

64. Freeman EE, Gange SJ, Muñoz B, West SK (2006) Driving status and risk of entry into long-term care in older adults. Am J Public Health 96:1254-1259

65. Marottoli RA, de Leon CFM, Glass TA, Williams CS, Cooney LM Jr, Berkman LF (2000) Consequences of driving cessation: decreased out-of-home activity levels. J Gerontol B Psychol Sci Soc Sci 55:S334-S340

66. Clark WL (2013) Long-term follow-up of intravitreal aflibercept injection (IAI) in patients with neovascular age-related macular degeneration. Presented at the AAO 2013 Annual Meeting; New Orleans, LA, USA, November 16-19, 2013

67. Clark WL, Boyer DS, Heier JS, Brown DM, Haller JA, Vitti R, Kazmi H, Berliner AJ, Erickson K, Chu KW, Soo Y, Cheng Y, Campochiaro PA (2016) Intravitreal aflibercept for macular edema following branch retinal vein occlusion: 52 -week results of the VIBRANT study. Ophthalmology 123:330-336

68. Chong V (2016) Ranibizumab for the treatment of wet AMD: a summary of real-world studies. Eye (Lond) 30:270-286

69. Prünte C, Fajnkuchen F, Mahmood S, Ricci F, Hatz K, Studnička J, Bezlyak V, Parikh S, Stubbings WJ, Wenzel A, Figueira J, RETAIN Study Group (2015) Ranibizumab 0.5 mg treat-andextend regimen for diabetic macular oedema: the RETAIN study. Br J Ophthalmol 100:787-795

70. Comparison of Age-related Macular Degeneration Treatments Trials (CATT) Research Group, Maguire MG, Martin DF, Ying GS, Jaffe GJ, Daniel E, Grunwald JE, Toth CA, Ferris FL 3rd, Fine SL (2016) Five-year outcomes with anti-vascular endothelial growth factor treatment of neovascular age-related macular degeneration: the comparison of age-related macular degeneration treatments trials. Ophthalmology. doi:10.1016/j.ophtha.2016.03.045

71. Comparison of Age-related Macular Degeneration Treatments Trials (CATT) Research Group, Martin DF, Maguire MG, Fine SL, Ying GS, Jaffe GJ, Grunwald JE, Toth C, Redford M, Ferris FL 3rd (2012) Ranibizumab and bevacizumab for treatment of neovascular age-related macular degeneration: two-year results. Ophthalmology 119:1388-1398

72. Oubraham H, Cohen SY, Samimi S, Marotte D, Bouzaher I, Bonicel P, Fajnkuchen F, Tadayoni R (2011) Inject and extend dosing versus dosing as needed: a comparative retrospective study of ranibizumab in exudative age-related macular degeneration. Retina 31:26-30

73. Brown DM, Heier JS, Clark WL, Boyer DS, Vitti R, Berliner AJ, Zeitz O, Sandbrink R, Zhu X, Haller JA (2013) Intravitreal aflibercept injection for macular edema secondary to central retinal vein occlusion: 1-year results from the phase 3 COPERNICUS study. Am J Ophthalmol 155:429-437

74. Korobelnik J-F, Holz FG, Roider J, Ogura Y, Simader C, SchmidtErfurth U, Lorenz K, Honda M, Vitti R, Berliner AJ, Hiemeyer F, Stemper B, Zeitz O, Sandbrink R, GALILEO Study Group (2014) Intravitreal aflibercept injection for macular edema resulting from central retinal vein occlusion: one-year results of the phase 3 GALILEO study. Ophthalmology 121:202-208

75. Bayer plc. EYLEA $40 \mathrm{mg} / \mathrm{mL}$ solution for injection in a vial summary of product characteristics. Bayer plc; Newbury, Berkshire, UK, November 2015, https://www.medicines.org.uk/ emc/medicine/27224 Last accessed December 28th, 2016

76. Goldstein M, Korobelnik J-F, Norenberg C, Zeitz O, VIEW investigators (2013) Subanalysis of visual acuity outcomes in the second year of VIEW studies. Presented at the ARVO 2013 Annual Meeting; Seattle, WA, USA, May 5-9, 2013 
77. Richard G, Monés J, Wolf S, Korobelnik J-F, Guymer R, Goldstein M, Norenberg C, Sandbrink R, Zeitz O (2015) Scheduled versus pro re nata dosing in the VIEW trials. Ophthalmology 122:2497-2503

78. Todorich B, Pepple K, Mruthyunjaya P (2012) Treatment of pigment epithelial detachments in age-related macular degeneration. Retin Physician 9:30-37

79. Lim JH, Wickremasinghe SS, Xie J, Chauhan DS, Baird PN, Robman LD, Hageman G, Guymer RH (2012) Delay to treatment and visual outcomes in patients treated with anti-vascular endothelial growth factor for age-related macular degeneration. Am J Ophthalmol 153:678-686

80. Rofagha S, Bhisitkul RB, Boyer DS, Sadda SR, Zhang K, SEVEN-UP Study Group (2013) Seven-year outcomes in ranibizumab-treated patients in ANCHOR, MARINA, and HORIZON: a multicenter cohort study (SEVEN-UP). Ophthalmology 120:2292-2299

81. Talks JS, Lotery AJ, Ghanchi F, Sivaprasad S, Johnston RL, Patel N, McKibbin M, Bailey C, Mahmood S, United Kingdom Aflibercept Users Group (2016) First-year visual acuity outcomes of providing aflibercept according to the VIEW study protocol for age-related macular degeneration. Ophthalmology 123:337-343

82. Manco-Johnson MJ, Abshire TC, Shapiro AD, Riske B, Hacker MR, Kilcoyne R, Ingram JD, Manco-Johnson ML, Funk S, Jacobson L, Valentino LA, Hoots WK, Buchanan GR, DiMichele D, Recht M, Brown D, Leissinger C, Bleak S, Cohen A, Mathew P, Matsunaga A, Medeiros D, Nugent D, Thomas GA, Thompson AA, McRedmond K, Soucie JM, Austin H, Evatt BL (2007) Prophylaxis versus episodic treatment to prevent joint disease in boys with severe hemophilia. N Engl J Med 357:535-544

83. Freedman MS, Comi G, De Stefano N, Barkhof F, Polman CH, Uitdehaag BM, Lehr L, Stubinski B, Kappos L (2014) Moving toward earlier treatment of multiple sclerosis: findings from a decade of clinical trials and implications for clinical practice. Mult Scler Relat Disord 3:147-155

84. Singer MA, Awh CC, Sadda S, Freeman WR, Antoszyk AN, Wong P, Tuomi L (2012) HORIZON: an open-label extension trial of ranibizumab for choroidal neovascularization secondary to agerelated macular degeneration. Ophthalmology 119:1175-1183

85. Heier JS, Boyer DS, Ciulla TA, Ferrone PJ, Jumper JM, Gentile RC, Kotlovker D, Chung CY, Kim RY, FOCUS Study Group (2006) Ranibizumab combined with verteporfin photodynamic therapy in neovascular age-related macular degeneration: year 1 results of the FOCUS Study. Arch Ophthalmol 124:1532-1542

86. Calvo P, Ferreras A, Wang Y, Devenyi RG, Lam WC, Brent MH (2013) Treat and extend vs. treat and observe in wet AMD patients treated with ranibizumab: a 3-year follow-up study. Presented at the AAO 2013 Annual Meeting; New Orleans, LA, USA, November 16-19, 2013

87. Hatz K, Prünte C (2016) Changing from a pro re nata treatment regimen to a treat and extend regimen with ranibizumab in neovascular age-related macular degeneration. Br J Ophthalmol 100(10):1341-1345. doi:10.1136/bjophthalmol-2015-307299

88. Epstein D, Amrén U (2016) Near vision outcome in patients with age-related macular degeneration treated with aflibercept. Retina 36(9):1773-1777
89. Regillo CD (2015) Prospective, multicenter investigation of aflibercept treat and extend therapy for neovascular age-related macular degeneration (ATLAS Study): two year results. Presented at the AAO 2015 Annual Meeting; Las Vegas, NV, USA, November 14-17, 2015

90. Muether PS, Hermann MM, Dröge K, Kirchhof B, Fauser S (2013) Long-term stability of vascular endothelial growth factor suppression time under ranibizumab treatment in age-related macular degeneration. Am J Ophthalmol 156:989-993

91. Fauser S, Schwabecker V, Muether PS (2014) Suppression of intraocular vascular endothelial growth factor during aflibercept treatment of age-related macular degeneration. Am J Ophthalmol 158:532-536

92. Fauser S, Muether PS (2016) Clinical correlation to differences in ranibizumab and aflibercept vascular endothelial growth factor suppression times. Br J Ophthalmol

93. Berg K, Hadzalic E, Gjertsen I, Forsaa V, Berger LH, Kinge B, Henschien H, Fossen K, Markovic S, Pedersen TR, Sandvik L, Bragadóttir R (2016) Ranibizumab or bevacizumab for neovascular age-related macular degeneration according to the Lucentis compared to Avastin study treat-and-extend protocol: two-year results. Ophthalmology 123:51-59

94. Mantel I, Deli A, Iglesias K, Ambresin A (2013) Prospective study evaluating the predictability of need for retreatment with intravitreal ranibizumab for age-related macular degeneration. Graefes Arch Clin Exp Ophthalmol 251:697-704

95. Muether PS, Droege KM, Fauser S (2014) Vascular endothelial growth factor suppression times in patients with diabetic macular oedema treated with ranibizumab. Br J Ophthalmol 98:179-181

96. Lorenz K (2014) Intravitreal aflibercept in CRVO: treatment interval extension based on visual and anatomic outcomes. Presented at the 5th World Congress on Controversies in Ophthalmology; Lisbon, Portugal, March 20-23, 2014

97. Heier JS, Brown DM, Chong V, Korobelnik J-F, Kaiser PK, Nguyen QD, Kirchhof B, Ho A, Ogura Y, Yancopoulos GD, Stahl N, Vitti R, Berliner AJ, Soo Y, Anderesi M, Groetzbach G, Sommerauer B, Sandbrink R, Simader C, Schmidt-Erfurth U, VIEW 1 and VIEW 2 Study Groups (2012) Intravitreal aflibercept (VEGF trap-eye) in wet age-related macular degeneration. Ophthalmology 119:2537-2548

98. Pfau M, Fassnacht-Riederle H, Becker MD, Graf N, Michels S (2015) Clinical outcome after switching therapy from ranibizumab and/or bevacizumab to aflibercept in central retinal vein occlusion. Ophthalmic Res 54:150-156

99. Khurana R (2015) Intravitreal aflibercept injection for previously treated macular edema associated with central retinal vein occlusions: twelve-month results from the NEWTON study. Presented at the AAO 2015 Annual Meeting; Las Vegas, NV, USA, November 14-17, 2015

100. Cohen SY, Dubois L, Tadayoni R, Fajnkuchen F, Nghiem-Buffet S, Delahaye-Mazza C, Guiberteau B, Quentel G (2009) Results of one-year's treatment with ranibizumab for exudative age-related macular degeneration in a clinical setting. Am J Ophthalmol 148: $409-413$ 